\title{
Análisis multicausal de Panel Cointegrado para el periodo 2000-2010 en el sistema microfinanciero boliviano
}

\author{
Multicasual analysis of Panel Cointegrado for the period \\ 2000-2010 in the microfinancial Bolivian system
}

\author{
Rolando Caballero Martínez* \\ Benigno Caballero Claure**
}

\section{Resumen}

Se analizan los efectos de las variables económico-financieras como el microcrédito, crédito de consumo, crédito hipotecario de vivienda, la tasa de interés real y el crecimiento económico en la cartera total del sistema microfinaciero de Bolivia. El enfoque se basa en la clasificación de la cartera total según tipo de crédito, de acuerdo a la clasificación de la autoridad de supervisión del sistema financiero de Bolivia (ASFI). Para poder analizar estos efectos se emplea como metodología econométrica, los modelos de datos de panel estático y dinámico (pooled regression, efectos fijos, efectos aleatorios y panel dinámico). Los resultados muestran, que todos los factores mencionados contribuyen a explicar la cartera total del sistema microfinanciero en Bolivia.

Palabras clave

- Instituciones microfinancieras

- Desarrollo Económico

- Modelos de Panel

\section{Abstract}

There are analyzed the effects of the economic-financial variables as the microcredit, credit of consumption, mortgage loan of housing, the rate of royal interest and the economic growth in the total portfolio of the system microfinaciero of Bolivia. The approach is based on the classification of the total portfolio according to type of credit, of agreement to the classification of the authority of supervision of the financial system of Bolivia (ASFI). To be able to analyze these effects it uses as methodology econométrica, the models of information of static and dynamic panel (pooled regression, fixed effects, random effects and dynamic panel). The results show, that all the mentioned factors help to explain the total portfolio of the microfinancial system in Bolivia.

\section{Keywords}

- Microfinancial Institutions

- Economic Development

- Models of Panel 
actualidad se atraviesa por algunas dificultades para el desarrollo de algunas actividades económicas, debido principalmente al entorno económico inestable, indudablemente las microfinanzas han sido y siguen siendo un éxito en Bolivia, a tal punto que se ha convertido en un ejemplo para los gobiernos e instituciones de muchos países.

Adicionalmente, se puede argumentar que a lo largo de casi dos décadas de sobresaliente historia, el desarrollo y el desempeño de las microfinanzas bolivianas han estado íntimamente ligados a la evolución de la economía del país.

Por lo anterior, cabe destacar que las microfinanzas en el caso boliviano, han sido y siguen siendo un poderoso instrumento de desarrollo económico, ya que varios investigadores mencionan que Bolivia a lo largo de los últimos años, ha demostrado que este instrumento puede convertirse en una poderosa herramienta, no de lucha contra la pobreza, sino de promoción del auténtico desarrollo económico que conlleva a la generación de empleo y riqueza, que incide especialmente en la población de rentas bajas. En consecuencia, el presente trabajo analiza cuales son los factores que han sido preponderantes para explicar, en los últimos años, los determinantes de la cartera total del sistema microfinanciero boliviano (ASFI) según tipo de crédito.

El presente artículo está estructurado de la siguiente manera: en el apartado 2 se describe la estrategia para modelar los determinantes de la cartera total $^{1}$ en el sistema microfinanciero en Bolivia, el tercer apartado desarrolla los resultados empíricos sobre el tema, el cuarto muestra las conclusiones de los resultados obtenidos.

A continuación se muestra de manera detallada la evolución gráfica de la cartera total de siete instituciones microfinancieras pertenecientes a la ASFI-Bolivia a lo largo del periodo 2000-2010.

${ }^{1}$ Los determinantes de la cartera total, serían según tipo de crédito y de acuerdo con la clasificación de la ASFI-Bolivia (http://www.asfi.gob.bo/Portals/0/Documentos/ Anuarios/2008/a08_90.pdf). 
Gráfica 1

Cartera total de las instituciones de microcrédito expresado

a través del tiempo. Por institución

(miles de bolivianos: eje de ordenadas)

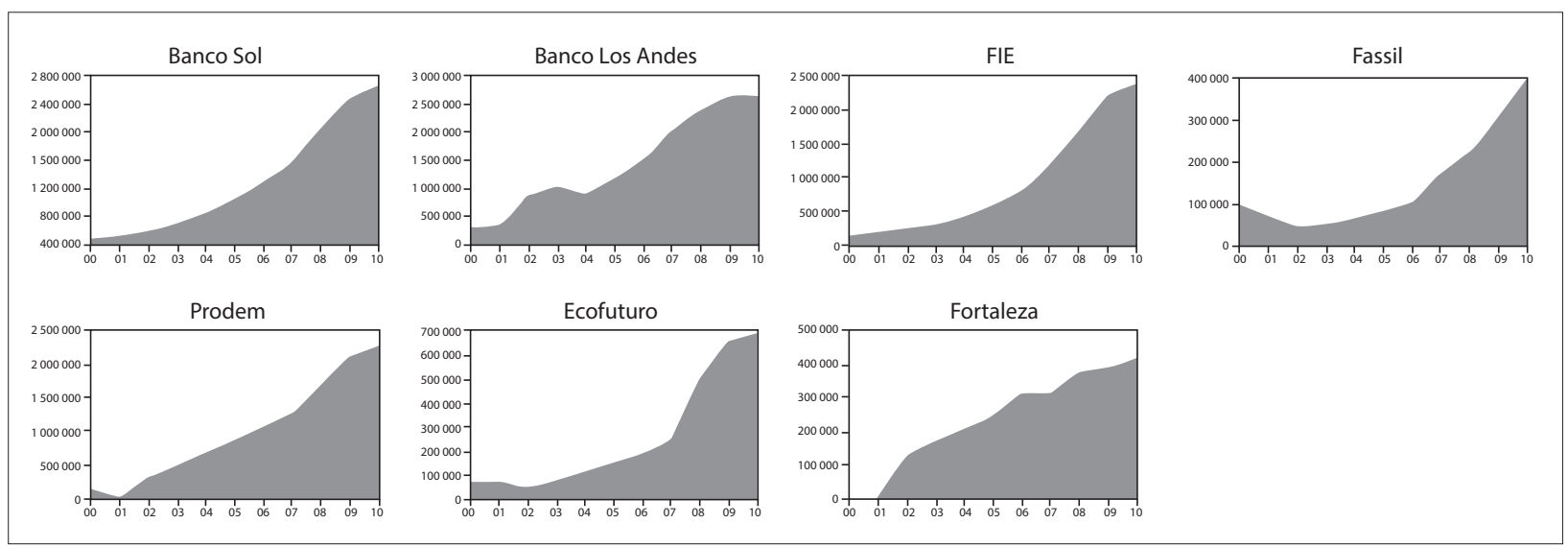

Fuente: elaboración propia de los autores.

Gráfica 2

Cartera total de las instituciones de microcrédito expresado

a través del tiempo

(miles de bolivianos: eje de ordenadas)

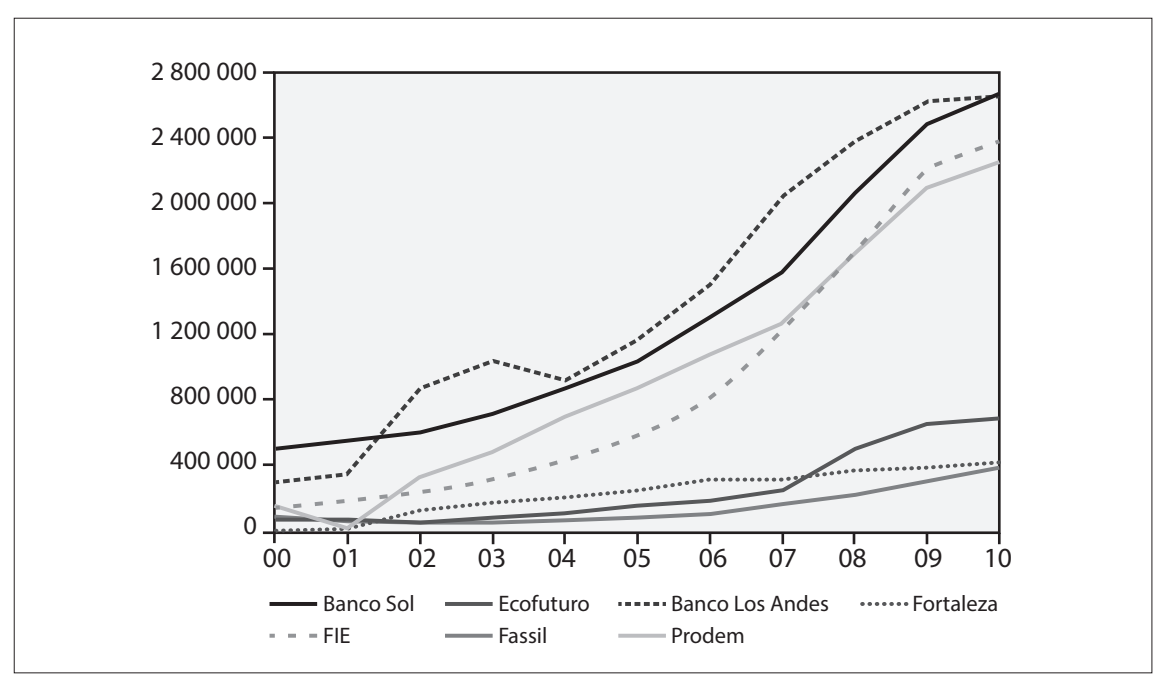

Fuente: elaboración propia de los autores. 
Economía Informa núm. 381 julio - agosto • 2013 ㅂ ㅁ |

\section{Factores que explican la Cartera Total según tipo de crédito}

La cartera de créditos es el activo más importante de las Entidades de Intermediación Financiera (EIF), debido a que constituye la principal fuente generadora de ingresos por lo que las operaciones de dicha cartera deben sustentarse adecuadamente en análisis objetivos de riesgo, y realizarse de acuerdo a estrategias, políticas y procedimientos establecidos por cada entidad financiera, pero debidamente aprobadas por el directorio $\mathrm{u}$ órgano equivalente, además de ajustarse a lo dispuesto en la Ley de Bancos y Entidades Financieras (LBEF).

A continuación daremos una breve descripción de la clasificación de la cartera total según tipo de crédito, de acuerdo a la clasificación de la Autoridad de Supervisión del Sistema Financiero (ASFI).

\section{a) Cartera Total (Tc)}

La cartera total expone el estado de cumplimiento de los créditos en las siguientes cuentas: cartera vigente, cartera vencida y cartera en ejecución, de acuerdo con las normas y criterios establecidos en la descripción de las respectivas cuentas.

\section{b) Microcrédito (cmic)}

Todo crédito otorgado a una persona natural o jurídica, o a un grupo de prestatarios, con el objeto de financiar actividades de producción, comercialización y servicios, cuya fuente principal de pago la constituye el producto de las ventas e ingresos generados por dichas actividades.

\section{c) Crédito Hipotecario de Vivienda (CHIP)}

Todo crédito otorgado a personas naturales destinado exclusivamente para:

- Adquisición de terreno para la construcción de vivienda

- Construcción de vivienda individual

- Compra, refacción, remodelación, ampliación, mejoramiento de vivienda individual o en propiedad horizontal

\section{d) Crédito de Consumo (ccon)}

Todo crédito concedido a una persona natural, con el objeto de financiar la adquisición de bienes de consumo o el pago de servicios, 
amortizable en cuotas sucesivas y cuya fuente principal de pago es el salario de la persona o ingresos provenientes de su actividad, adecuadamente verificados. Esta definición incluye las operaciones realizadas a través del sistema de tarjetas de crédito de personas naturales.

\section{e) La Tasa de Interés Real (TIAE)}

La tasa de interés real es una de las variables fundamentales para explicar las relaciones que se establecen entre la esfera productiva y la financiera, representa uno de los canales de transmisión más importantes de la política monetaria, asociado al coste de financiamiento, y permite además identificar la relación que se establece entre la tasa de interés nominal y la inflación (Walsh, 2000).

\section{f) Crecimiento Económico}

El crecimiento económico es una de las metas de toda sociedad, implica un incremento notable de los ingresos, y de la forma de vida de todos los individuos de una sociedad. Existen muchas maneras o puntos de vista desde los cuales se mide el crecimiento de una sociedad, se podría tomar como ejes de medición la inversión, el nivel de consumo, las políticas gubernamentales, o las políticas de fomento al ahorro.

\section{Formulación del problema de investigación}

Tomando en cuenta el creciente desarrollo de las actividades económicas de las micro y pequeña empresas y el desarrollo de productos financieros de acuerdo a las necesidades de cada región orientados a los sectores económicos y aprovechando el conocimiento de los segmentos del mercado en el sistema financiero boliviano, se observa que existe un conglomerado de variables que influyen en el comportamiento de la cartera total.

El presente trabajo se centralizara en la investigación la cartera total de las siete entidades financieras de microcrédito que son: Banco Solidario, Banco los Andes, FIE, Prodem, Ecofuturo, Fortaleza y Fassil, que viene influido por varias variables de las cuales se considerará las de mayor relevancia como son el crédito comercial, el crédito hipotecario de vivienda, el microcrédito, el crédito de consumo y la tasa de interés activa en moneda extranjera en el comportamiento de la economía, todo esto mediante datos longitudinales de estas variables en un modelo econométrico, de tal mane- 
ra que los resultados nos permitan evaluarlos para luego obtener conclusiones, para que en base a ellas se proceda a una eficiente toma de decisiones de las entidades financieras. Es en ese sentido que se presenta la siguiente formulación del problema:

¿Cuál es el impacto y la significancia del microcrédito, crédito comercial, crédito de consumo, crédito hipotecario de vivienda, tasa de interés en moneda extranjera y crecimiento económico sobre la cartera total de las siete entidades microfinancieras reguladas?

\section{Justificación teórica}

La literatura para el caso de Bolivia muestra de manera relativa a través de algunos trabajos de investigación los distintos determinantes de la cartera total para el sistema financiero boliviano.

Más propiamente lo que se hace es analizar la interacción que existe entre las variables financieras y económicas con relación a la cartera total, es el caso por ejemplo de Gonzáles y Rodríguez (2003) que se focalizan en la interacción de las variables macroeconómicas y financieras sobre las microfinanzas en Bolivia y para ello hacen uso de una muestra que va desde: bancos comerciales, mutuales, cooperativas e instituciones microfinancieras, etcétera.

El análisis de Gonzáles y Ramírez (2003), se focaliza esencialmente al análisis de las variables desde un punto de vista gráfico, se muestra la evolución cronológica de las variables a través del tiempo y su interacción con las demás variables, se llega a la conclusión de que las variables macro y financieras, así como las necesidades crecientes de la población boliviana en su conjunto, son importantes para en buen funcionamiento de las microfinanzas en Bolivia.

Otro trabajo es el de Ynajara Ramírez (2000), dicho trabajo de investigación tiene por objeto difundir la experiencia en Bolivia de lo que se ha demostrado que puede ser un poderoso instrumento de desarrollo: las microfinanzas, además, el autor comenta que los éxitos alcanzados por Bolivia en este campo a lo largo de los últimos años demuestran que este instrumento puede convertirse en una poderosa herramienta no sólo contra la pobreza, sino también para la generación de empleo y riqueza en la población de rentas bajas.

El análisis de Ynajara Ramírez (2000) es un tanto más laxo con relación al de González y Ramírez (2003), ya que sólo se focaliza al análisis de la 
variables microfinancieras desde un punto de vista teórico y tomando como punto de partida la evidencia empírica, pero carece de análisis gráfico de las variables y la interacción analítica de las mismas, es decir, se llega a conclusiones teóricas relevantes en el sistema financiero de Bolivia, tomando como base los aspectos teóricos del mismo, pero careciendo de un fuerte análisis cuantitativo.

Por lo tanto se puede argumentar que una vez revisado varios documentos de investigación al respecto, todos estos tienen un común denominador: las microfinanzas en Bolivia son un instrumento importante para el desarrollo económico en el país, una útil herramienta de lucha contra la pobreza, promoción de auténtico desarrollo con generación de empleo y riqueza, especialmente en la población de rentas bajas y, en ese sentido, es muy importante conocer que variables -desde el punto de vista de las microfinanzas en Bolivia y la evidencia empírica de la misma- son las que tienen mayor grado de interacción entre ellas mismas y cuáles son las que tienen mayor impacto sobre la cartera total del sistema financiero en Bolivia para luego realizar el análisis y la toma de decisiones en política que ayuden a mejorar el entorno económico. Es en ese sentido, revisada la literatura al respecto, se puede decir que los trabajos mencionados, si bien hacen un aporte teórico muy importante a las microfinanzas en Bolivia y en muchos casos presentan estudios de análisis grafico longitudinal de las variables en estudio, no presentan el impacto y la significancia que existe entre las variables. ${ }^{2}$

\section{Resultados empíricos}

En esta sección se describe la estrategia para modelar los determinantes de la cartera total del sistema microfinanciero en Bolivia, tomando en cuenta que las variables que se utilizan en el estudio para siete instituciones de microcrédito tienen una frecuencia anual y fueron obtenidas del Banco Central de Bolivia (всв), la Unidad de Análisis de Políticas Económicas y Sociales (UDAPE), los bancos de datos estadísticos de la Autoridad de Supervisión del Sistema Financiero de Bolivia (ASFI) y ASOFIn Bolivia.

La metodología que se aplicará es deductiva, es decir, se empezara estimando varios modelos de datos panel, tales, como: efectos fijos, efectos

${ }^{2}$ Los datos de las variables están expresados en miles de bolivianos y fueron tomados de la página web estadística del anuario estadístico de la ASFI-BoliviA. (www.asfi.gob. bo/Estad\%C3\%ADsticas/IntermediacionFinanciera/AnuarioEstad\%C3\%ADstico). La tasa de interés real y el crecimiento económico fueron obtenidos de la base de datos del Banco Central de Bolivia (www.bcb.gob.bo/?q=estadisticas/semanales). 
aleatorios, modelo plano y panel dinámico, con y sin errores estándar robustos, para luego ir descartando uno por uno, a través de distintas pruebas de selección de modelos. ${ }^{3}$

El modelo propuesto ${ }^{4}$ en este trabajo para analizar los efectos sobre la cartera es:

$$
\mathrm{CT}_{\mathrm{IT}}=\psi_{1}+\psi_{2} \mathrm{CMIC}_{I T}+\psi_{4}+\psi_{5} \mathrm{CCOM}_{I T}+\psi_{6} T I A E_{I T}+\xi_{I T}
$$

Donde:

$\psi_{1}$ :Término constante o intercepto

$\mathrm{CT}_{\mathrm{TT}}$ : Cartera total del sistema microfinanciero boliviano

$\mathrm{CMIC}_{\mathrm{IT}}$ : Microcrédito del sistema FFPs de Bolivia

CHIP $_{\text {IT }}$ Crédito hipotecario de vivienda del sistema FFPs de Bolivia

$\mathrm{CCON}_{\mathrm{IT}}$ : Crédito de consumo del sistema FFP de Bolivia

$\mathrm{CCOM}_{\mathrm{IT}}$ : Crédito comercial del sistema FFPs de Bolivia

$\mathrm{TIAE}_{\mathrm{TT}}$ : Tasa de interés activa en moneda extranjera del sistema FFPs de Bolivia

$\xi_{I T}$ : Error idiosincrásico qu recoge todas la variables que no estan implicadas en el modelo.

Donde: $\forall I=I, \ldots, T \forall I=I \ldots$, que es el identificador de sección cruzada (núm. FFP de Bolivia que son 7).

Donde: $\forall T=I, \ldots, T$ que es el identificador de series de tiempo (núm. de periodos de estudio de 2000 al 2005 .

$\xi \approx N I I D(o, \Sigma)$ se refiere al error idiosincrásico que sigue un proceso de ruido blanco.

Ruido blanco implica que el error idiosincrásico tiene media cero y varianza constante.

La base del modelo consiste en suponer que la dinámica de la cartera total de las instituciones que pertenecen a la Asofin Bolivia, es afectada por todos los elementos de la cartera total según clasificación de crédito, como: microcrédito, crédito comercial, crédito de consumo, crédito hipotecario de vivienda, tasa de interés en mE y crecimiento económico de Bolivia.

\footnotetext{
${ }^{3}$ Por lo tanto a través de distintas pruebas de hipótesis se concluye que el mejor modelo entre el Pooled, Efectos fijos y Efectos Aleatorios, es el modelo de efectos fijos que mejor se ajusta y por lo mismo, el que mejor describe la dinámica de la cartera total en las instituciones pertenecientes a ASFI-BOLIVIA, sin embargo por razones de espacio no se muestran en este documento las estimaciones iniciales del modelo Pooled, Efectos Fijos y Efectos Aleatorios. Sin embargo, si alguien necesitara las estimaciones de los modelos iniciales con gusto los autores las proporcionaran.

${ }^{4}$ La prueba de Hausman, para elegir entre un modelo de efectos fijos y aleatorios se encuentra en el anexo 2.
} 


\section{Cuadro 1}

(Efectos Fijos ${ }^{5}$ con y sin corrección de Heterocedasticidad)

Primer modelo final, método de mínimos cuadrados generalizados factibles (FGLS) para corregir heterocedasticidad en el modelo de efectos fijos con efectos temporales y de espacio

\begin{tabular}{|c|c|c|c|c|c|c|}
\hline \multirow[b]{2}{*}{ Variables } & \multicolumn{3}{|c|}{$\begin{array}{c}\text { Modelo Efectos Fijos } \\
\text { (con Corrección de Heterocedastidad) }\end{array}$} & \multicolumn{3}{|c|}{$\begin{array}{l}\text { Modelo Efectos Fijos con } \\
\text { Errores Estándar Robustos }\end{array}$} \\
\hline & Coeficiente & $\begin{array}{l}\text { Error } \\
\text { Estándar }\end{array}$ & \begin{tabular}{c|} 
Valor de \\
Probabilidad
\end{tabular} & Coeficiente & $\begin{array}{l}\text { Error } \\
\text { Estándar }\end{array}$ & $\begin{array}{c}\text { Valor de } \\
\text { Probabilidad }\end{array}$ \\
\hline CONSTANTE & -0.145 & 0.542 & 0.489 & 1.458 & 0.652 & 0.117 \\
\hline CHIP & 0.015 & 0.022 & 0.384 & 0.030 & 0.015 & 0.133 \\
\hline CMIC & 0.951 & 0.048 & 0.000 & 0.972 & 0.028 & 0.000 \\
\hline $\mathrm{CCON}$ & 0.020 & 0.027 & 0.951 & 0.006 & 0.022 & 0.783 \\
\hline CREC & 0.304 & 0.085 & 0.003 & 0.143 & 0.058 & 0.011 \\
\hline $\mathrm{CCOM}$ & 0.010 & 0.018 & 0.914 & -0.001 & 0.012 & 0.912 \\
\hline TIAE & & & & -5.811 & 1.721 & 0.008 \\
\hline DUM & -12.654 & 0.801 & 0.000 & -13.100 & 0.332 & 0.000 \\
\hline \multicolumn{4}{|l|}{ Efectos Fijos } & & & \\
\hline Banco los Andes & -0.237 & 0.231 & 0.132 & & & \\
\hline FÍE & -0.075 & 0.286 & 0.733 & & & \\
\hline Prodem & -0.446 & 0.272 & 0.049 & & & \\
\hline Ecofuturo & -0.598 & 0.273 & 0.012 & & & \\
\hline Fortaleza & 0.037 & 0.310 & 0.892 & & & \\
\hline Fassil & -0.321 & 0.247 & 0.095 & & & \\
\hline 2001 & 0.111 & 0.288 & 0.832 & & & \\
\hline 2002 & 0.029 & 0.267 & 0.972 & & & \\
\hline 2003 & 0.035 & 0.258 & 0.890 & & & \\
\hline 2004 & -0.293 & 0.219 & 0.041 & & & \\
\hline 2005 & -0.367 & 0.223 & 0.011 & & & \\
\hline 2006 & 0.179 & 0.218 & 0.135 & & & \\
\hline 2007 & 0.543 & 0.236 & 0.025 & & & \\
\hline 2008 & 0.576 & 0.227 & 0.032 & & & \\
\hline 2009 & 0.783 & 0.274 & 0.011 & & & \\
\hline 2010 & 0.552 & 0.260 & 0.035 & & & \\
\hline \multicolumn{7}{|c|}{ Estadísticos de Diagnostico } \\
\hline \multicolumn{3}{|c|}{$\begin{array}{c}\text { Modelo Efectos Fijos } \\
\text { (Con Corrección de Heterocedastidad) }\end{array}$} & \multicolumn{4}{|c|}{$\begin{array}{c}\text { Modelo Efectos Fijos } \\
\text { con Errores Estándar Robustos }\end{array}$} \\
\hline \multicolumn{3}{|c|}{$\begin{array}{l}\mathrm{R}^{2}=0.9729 \\
\text { Estadístico } \mathrm{F}=2410.97\end{array}$} & \multicolumn{4}{|c|}{$\begin{array}{l}\mathrm{R}^{2}=0.9526 \\
\text { Valor p de } \mathrm{F}=0.0000 \\
\text { Estadistico } \mathrm{F}=206.99 \\
\text { Prueba } \mathrm{F} F(6,62)=\quad 1.79 \\
\text { Valor de Probabilidad } \mathrm{F}=0.0860\end{array}$} \\
\hline
\end{tabular}

Cabe hacer notar que la variable tasa de interés activa en moneda extranjera (TIAE) no tiene coeficiente en el modelo de efectos fijos con corrección de heterocedasticidad, debido a que es eliminado automáticamente al estimarlo con el software, por dos motivos: problemas de multicolinealidad y para la posible iteración del modelo y su respectiva solución.

Fuente: elaboración propia de los autores.

\footnotetext{
${ }^{5}$ Cabe recalcar que las pruebas de diagnóstico del modelo de efectos fijos se encuentran en el anexo 1.
} 
El método de mínimos cuadrados generalizados factibles (FGLS), ${ }^{6}$ para corregir heterocedasticidad es un primer método que soluciona el problema de heterocedasticidad en el modelo de efectos fijos y vendría a ser el primer modelo final que se utilizaría para la interpretación de resultados.

En ambos modelos se puede advertir lo siguiente:

- Que todas las variables de manera conjunta explican de manera significativa a la variable dependiente que es la cartera total y cuya afirmación se apoya en el hecho de que el valor de probabilidad de: Prob. $>$ chi $^{2}=0.0000<0.10$ y por lo tanto ello demuestra la significancia global de todas las variables.

- Gue todas las variables de estudio tienen los signos esperados a priori.

- En ambos modelos se puede advertir que existe tres variables que son significativas de manera individual para poder explicar el comportamiento de la cartera total de las entidades pertenecientes a Asofin-Bolivia, estas variables significativas son: microcrédito (cmic), crecimiento económico (crec), tasa de interés activa en moneda extranjera (tiae) y la dumy (dum) y son significativas ya que su valor de probabilidad (prob) es menor a 0.10, mientras que las variables no significativas y que por lo mismo no explican de manera significativa la cartera total (Tc) de las entidades de Asofin son: crédito hipotecario de vivienda, el crédito de consumo (ccom) y el crédito comercial.

Respecto a los efectos diferenciales entre las distintas unidades de sección cruzada (distintas instituciones de microcrédito) se tiene la siguiente especificación:

\begin{tabular}{|c|c|}
\hline Banco los Andes & FIE \\
\hline Prodem & Ecofuturo \\
\hline Fortaleza & Fassil \\
\hline
\end{tabular}

La incorporación de variables dicotómicas para cada institución de microcrédito permite modelar características de las unidades transversales que no cambian en el tiempo, pero sí puede existir diferencia entre las siete ins-

6 Véase: http://www.ulpgc.es/hege/almacen/download/8/8071/capitulo_7.pdf [20/03/2011]. 
tituciones de microcrédito, en el modelo se puede advertir que no aparece la Dummy que corresponde al Banco Sol y ello es debido a que no se quiere incurrir en problemas de colinealidad (Caballero Benigno, 2003; Mendoza y Quintana, 2008).

De las seis dummis, se puede advertir que las significativas corresponden a: Prodem $=-0.446$, Ecofuturo $=-0.598$ y Fassil $=-0.321$ y son significativas ya que su valor de probabilidad es $<0.10$ y al ser significativas, señala que existe diferencia de estas tres instituciones de microcrédito. Respecto a las otras cuatro instituciones de microcrédito en estudio y como su signo es negativo, también señalaría que estas tres instituciones de microcrédito (Prodem, Ecofuturo y Fassil) son poco sensibles al efecto de la cartera total.

Ahora con relación al efecto fijo para las variables dicotómicas en el tiempo también es posible agregar variables dicotómicas temporales a nuestro modelo, es decir, una para cada año en la muestra, que capturen eventos comunes a todas las instituciones de microcrédito durante un período $\mathrm{u}$ otro, como una gran depresión, problemas políticos, corridas bancarias, etcétera.

Además las variables dicotómicas de efectos temporales permitirán controlar, por aquellos eventos, a los que fueron sujetos todas las instituciones de microcrédito en un año dado.

Y los efectos temporales correspondientes a cada año son:

\begin{tabular}{|l|l|l|l|}
\hline 2001 & 2002 & 2003 & 2004 \\
\hline 2005 & 2006 & 2007 & 2008 \\
\hline & 2009 & 2010 & \\
\hline
\end{tabular}

De manera automática se puede notar que el modelo no toma en cuenta las dummys para el Banco Sol y tampoco para el primer año en estudio que es el 2000, ello para no incurrir en la trampa de la variable dicotómica (multicolinealidad perfecta).

De las diez dummys temporales, se puede advertir que las significativas corresponden a: $2004=-0.293,2005=-0.367,2007=0.543,2008=$ $0.576,2009=0.783$ y $2010=0.552$ y son significativas ya que su valor de probabilidad es < 0.10 y al ser significativas, señalaría que en esos años ha sucedido hechos socio-económicos muy importantes que han afectado la dinámica de la cartera total de las entidades pertenecientes a Asofin.

Más específicamente en los años 2004 y 2005 (2004 = -0.293 y 2005 $=-0.367$ ) comienza en Bolivia y también en América Latina un contexto 
externo favorable traducido en buenos precios de los commodities (materias primas) y además en el caso boliviano empezaba una crisis política de Estado muy fuerte tras la salida abrupta del gobierno de Sánchez de Lozada y como las dummys de los años 2004 y 2005 tienen coeficientes negativos, ello afirmaría que debido al contexto externo favorable traducido en buenos precios de las materias primas que Bolivia exporta y también debido al comienzo de una crisis política fuerte, disminuye la sensibilidad de la cartera total, es decir en términos más concretos la cartera total de las entidades pertenecientes a ASOFIN en los años 2004 y 2005 es poco sensible ante el comienzo de una crisis política y ante el contexto externo favorable.

Pero con respecto a los años 2007, 2008, 2009 y $2010(2007=0.543$, $2008=0.576,2009=0.783$ y $2010=0.552$ ) comienza en Bolivia y también en América Latina dos hechos muy importantes:

- Una desaceleración del crecimiento económico a nivel mundial, fruto de la crisis de las hipotecas subprime que es una crisis financiera que se extiende por los mercados financieros, principalmente, a partir del jueves 9 de agosto de 2007 en Estados Unidos y como representa 25\% del PIB mundial, la desaceleración de su crecimiento económico provoca iguales consecuencias a nivel mundial en un grado menor y Bolivia también es afectada por ello, lo que en términos de las ciencias sociales se conoce como la hipótesis del decoupling.

- Fuertes presiones inflacionarias se reflejan en toda la región latinoamericana, comienzan en 2007, para el año 2008 Bolivia termino siendo el segundo país de América Latina con una tasa de inflación elevada de 14\% anual.

El coeficiente de determinación (R-cuadrado) tiene un valor de: 0.9729, afirmaría que $97.29 \%$ de las variaciones de la cartera total de las siete instituciones de microcrédito vienen explicadas por las variables que se toman en cuenta en el presente estudio (CHIP, CMIC, CCON, CREC, TIAE y CCOM) y que solo $2.71 \%$ de las variaciones de la cartera total vienen explicadas por variables que no se toman en cuenta en el estudio, como el R-cuadrado: 0.9729 está muy próximo a 1, implica un excelente ajuste del modelo de efectos fijos. 


\section{Contraste de diferentes interceptos por grupos en el Modelo de Efectos Fijos}

Hipótesis nula: Los grupos tienen un intercepto común

Estadístico de contraste: $\mathrm{F}(6,63)=1.86897$

Con valor de probabilidad $=\mathrm{P}(\mathrm{F}(6,63)>1.86897)=0.100154$

Como el valor de probabilidad es: $0.100154>0.05$, implica que se acepta la hipótesis nula (Ho), es decir, que las siete instituciones de microcrédito pertenecientes a ASOFIN tienen un intercepto común, si bien existe diferencias entre ellas en cuanto a nichos de mercado, servicios al cliente, focalización de servicios a las zonas urbana o rural, estas diferencias son mínimas y que en promedio las distintas instituciones de microcrédito pertenecientes a ASOFIN-BOLIVIA tienen las mismas características.

\section{Gráfica 3}

\section{Simulación del Modelo de Efectos Fijos}

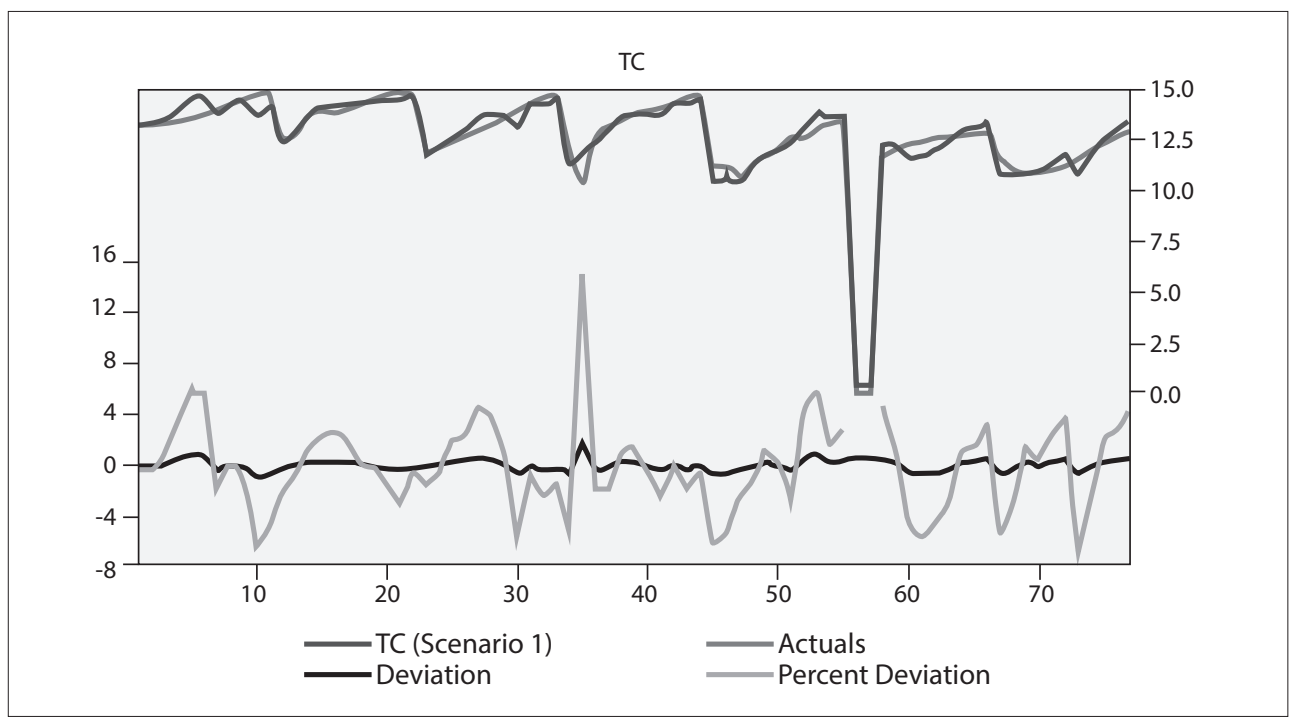

Fuente: elaboración propia de los autores.

La simulación del modelo de efectos fijos, confirma el buen ajuste del modelo de efectos fijos a los datos y por lo tanto como un excelente proceso generador de información de datos panel. 


\section{Evidencia dinámica de los determinantes de cartera total de Bolivia: estimación del Panel Dinámico}

Como este es el tercero de los modelos finales, se puede advertir que los signos de cada uno de los regresores son los esperados a priori, además se puede advertir que los instrumentos están bien definidos, debido a que la prueba de Sargan tiene un valor de probabilidad de $0.273>0.05$. También se puede afirmar que el modelo de Panel Dinámico tiene autocorrelación de primer orden, pero no así de segundo orden que es típico y aceptable en modelos expresados en diferencias.

\section{Cuadro 2}

\begin{tabular}{|c|c|c|c|}
\hline \multirow{2}{*}{ Variables } & \multicolumn{3}{|c|}{ Modelo panel dinámico } \\
\hline & Coeficiente & Error estándar & Valor de probabilidad \\
\hline Constante & 0.165 & 0.034 & 0.000 \\
\hline TC $(-1)$ & 0.085 & 0.055 & 0.049 \\
\hline CHIP & 0.008 & 0.016 & 0.601 \\
\hline CMIC & 0.892 & 0.077 & 0.000 \\
\hline $\mathrm{CCON}$ & 0.003 & 0.030 & 0.912 \\
\hline CREC & 0.076 & 0.038 & 0.003 \\
\hline $\mathrm{CCOM}$ & 0.046 & 0.021 & 0.035 \\
\hline TIAE & -6.664 & 3.177 & 0.041 \\
\hline DUM & -12.094 & 0.984 & 0.000 \\
\hline Banco de los Andes & -0.141 & 0.046 & 0.004 \\
\hline FIE & -0.083 & 0.045 & 0.077 \\
\hline Prodem & -0.041 & 0.072 & 0.571 \\
\hline Ecofuturo & -0.213 & 0.050 & 0.000 \\
\hline Fortaleza & -0.218 & 0.088 & 0.017 \\
\hline Fassil & -0.198 & 0.028 & 0.000 \\
\hline \multicolumn{4}{|c|}{$\begin{array}{l}\text { Prueba de Wald (inclusión de Dummy) }=1546 \text { : Valor de probabilidad }=0.000 \\
\text { Prueba de Sargan }=51.32 ; \text { Valor de probabilidad }=0.273 \\
\text { Prueba AR }(1)=0.043 \\
\text { Prueba AR }(2)=0.99\end{array}$} \\
\hline
\end{tabular}

Fuente: elaboración propia de los autores. 
Respecto a la variable que captura la persistencia del regresado $\left(\mathrm{TC}^{-1}\right)$ que es la cartera total rezagada en un periodo y cuyo impacto de corto plazo es: 0.085, una forma de interpretarlo sería que si la cartera total en el periodo actual aumenta en un punto porcentual, la cartera total en el próximo periodo aumentaría en $0.08 \%$, además que esta variable es significativa a corto plazo para explicar la Tc de manera individual, y ello confirmaría que la cartera total de las siete instituciones de microcrédito pertenecientes a ASOFIN tiene un comportamiento de inercia en la cartera total, es decir que el comportamiento de la cartera total de periodos pasados afecta al comportamiento de la cartera total en el periodo presente y futuro.

Por otro lado de las seis dummis de las instituciones de microcrédito, se puede advertir que cinco dummis son significativas y corresponden a: Banco los Andes, FIE, Ecofuturo, Fortaleza y Fassil, al ser significativas nos diría que existen diferencias de estas cinco instituciones de microcrédito respecto a Prodem.

\section{Gráfica 4}

Simulación del Panel Dinámico Valor Observado (punteado) y Valor Pronosticado por Panel Dinámico (continuo)

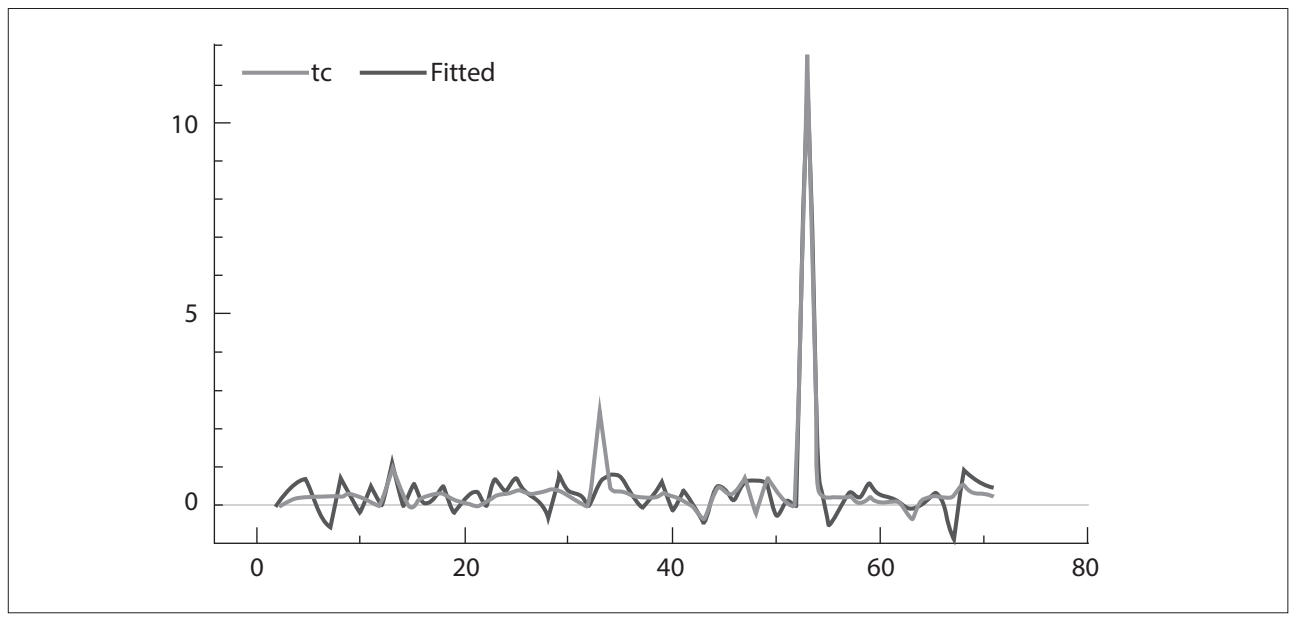

Fuente: elaboración propia de los autores. 


\section{Gráfica 5}

Residuales del Panel Dinámico y Función de Autocorrelación (ACF) y Función de Autocorrelación Parcial (PACF)
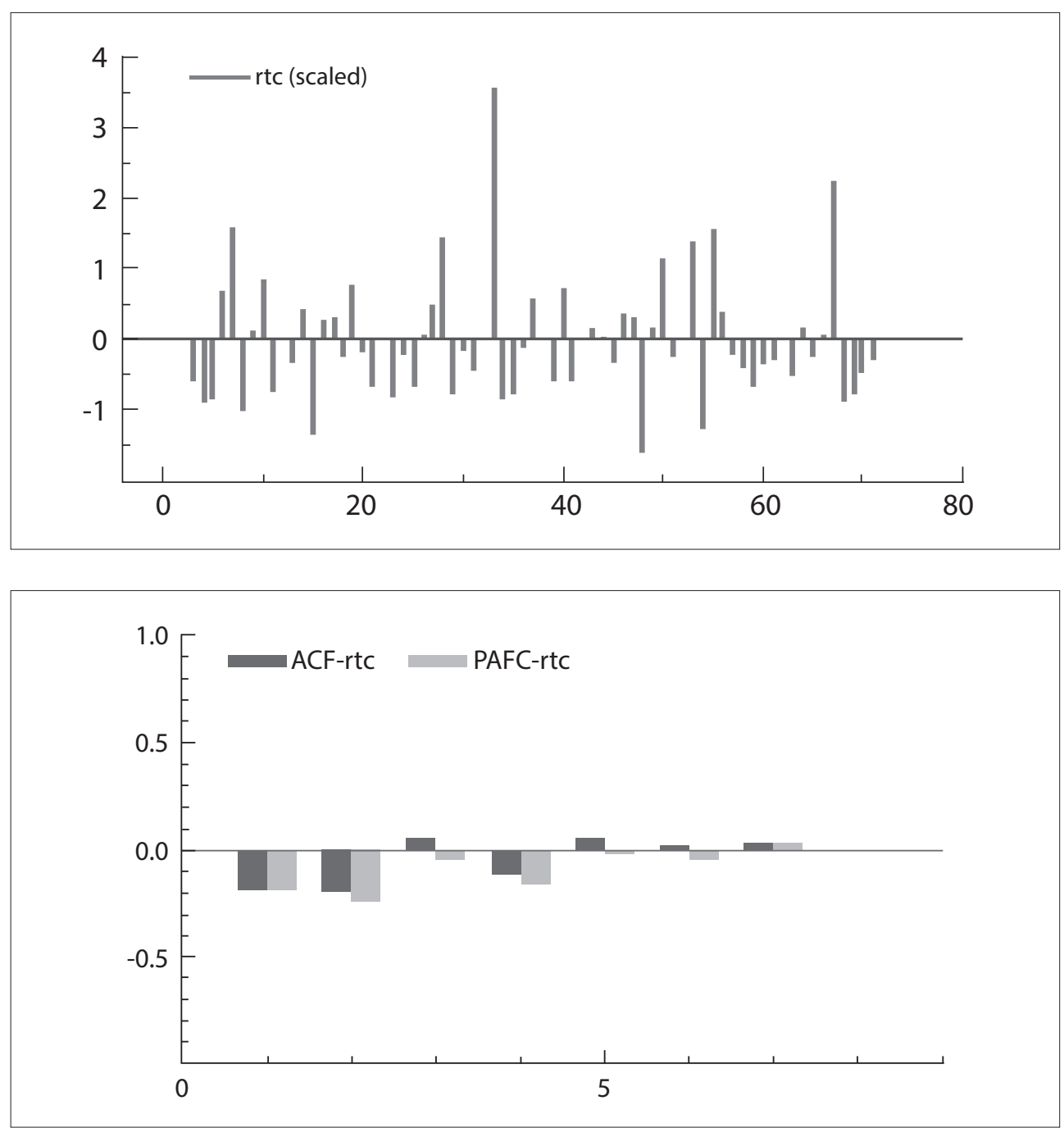

Fuente: elaboración propia de los autores.

Por último la gráfica 5 nos muestra el excelente ajuste del modelo de panel dinámico a los datos y por lo tanto confirma que es candidato a ser utilizado como un buen generador de información. 


\section{Prueba de Panel Cointegrado}

La Cointegración ${ }^{7}$ corresponde a una o más relaciones de largo plazo entre variables que tienen raíz unitaria I (1) y el hecho de que las variables estén cointegradas implica que presentan las mismas tendencias estocásticas y que no se incurriría en problemas de regresión espuria (falsa) ${ }^{8}$ lo cual es muy deseable (Loria, 2009).

\section{Gráfica 6 Análisis gráfico de Cointegración}

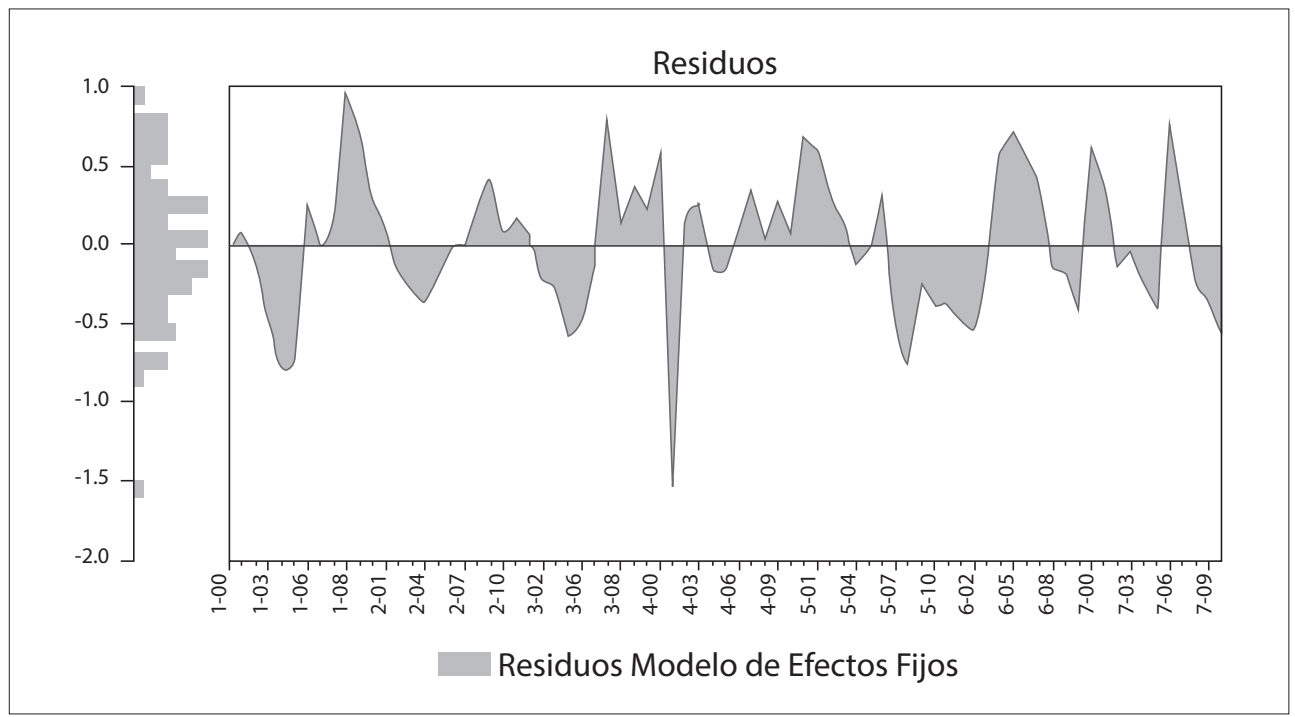

Fuente: elaboración propia de los autores.

\footnotetext{
${ }^{7}$ Una serie es estacionaria si su distribución de probabilidad no depende del tiempo, lo que implica una media y varianza uniformes en el tiempo. Muchas de las series del mundo real, tanto en economía como en ingeniería, resultan o parecen ser no estacionarias (una vez eliminados los posibles componentes deterministas de la serie). El tipo de series no estacionarias más habituales son las series integradas de orden 1, cuyas primeras diferencias son estacionarias. El tratamiento estadístico de estas series exige precauciones, porque los métodos de inferencia habituales pueden no ser válidos. Así, por ejemplo, los estadísticos de contraste de regresión no siguen las distribuciones estándar correspondientes a procesos estacionarios, lo que da lugar a las denominadas regresiones espurias (con frecuencia se identifican como significativas relaciones que no existen realmente).

${ }^{8}$ Véase Cameron, C. y P. Trivedi (2005), Microeconometrics-Methods and Applications, USA: Cambridge-Press, p. 112.
} 
En la gráfica de los residuos del modelo de efectos fijos, se puede advertir de que existe un proceso de reversión a su media y que no tiene patrones sistemáticos (tendencia positiva o negativa), por lo tanto, desde el punto de vista gráfico, se afirma que los residuos son estacionarios o dicho de otra forma que existe cointegración en las variables en estudio. ${ }^{9}$

\section{Cuadro 3}

\section{Pruebas formales de Cointegración}

\begin{tabular}{|c|c|c|c|c|}
\hline \multicolumn{5}{|c|}{$\begin{array}{l}\text { Prueba de Cointegración de Kao } \\
\text { Series: TC, CHIP, CMIC, CCON, CCOM, CREC } \\
\text { Muestra: 2000-2010 } \\
\text { Observaciones incluidas: } 77 \\
\text { Hipótesis nula: No cointeración } \\
\text { Supuesto de tendencia: No tendencia determinista }\end{array}$} \\
\hline & & & Estadístico & Probabilidad \\
\hline $\mathrm{ADF}$ & & & -3.753786 & 0.0001 \\
\hline Residual variance & & & 1.495177 & \\
\hline HAC variance & & & 0.602299 & \\
\hline \multicolumn{5}{|c|}{$\begin{array}{l}\text { Prueba de cointegración Pedroni } \\
\text { Series: tc, chip, cmic, ccon, ccom, crec } \\
\text { Inclusión de secciones cruzadas: } 6 \text { (1 dropped) } \\
\text { Observaciones incluidas: } 77 \\
\text { Hipótesis nula: No cointeración } \\
\text { Supuesto de tendencia: No intercepto y no tendencia determinista }\end{array}$} \\
\hline & Estadístico & Probabilidad & Estadístico ponderado & Probabilidad \\
\hline Panel v-estadístico & -2.592020 & 0.0139 & -2.745606 & 0.0092 \\
\hline Panel RHO-estadístico & 1.880765 & 0.0680 & 1.889637 & 0.0669 \\
\hline Panel PP-estadístico & -7.942772 & 0.0000 & -7.536684 & 0.0000 \\
\hline Panel ADF-estadístico & -3.720112 & 0.0004 & -3.171949 & 0.0026 \\
\hline
\end{tabular}

Fuente: elaboración propia de los autores.

Como los valores de probabilidad (Prob.) del Test de Kao y Pedroni en sus distintas variantes son menores a 0.10, se acepta la Hipótesis alternativa (Ha), Por lo tanto a través de las pruebas de cointegración de Kao y Pedroni, se puede afirmar que las series de datos panel en estudio están cointegradas.

${ }_{9}^{9}$ Gujarati, D. (2005), Econometría Básica, México, DF: McGraw Hill, pp. 795-799. 


\section{Conclusiones}

Se puede afirmar que ambos modelos finales, correspondientes a (Efectos Fijos y Panel Dinámico), antes de ser mutuamente excluyentes, son colectivamente exhaustivas (se complementan). En ambos modelos las variables explicativas tienen los signos esperados. Por otro lado también en ambos modelos (Efectos Fijos y Panel Dinámico) se confirma que las variables que explican de manera significativa la cartera total son: microcrédito, crecimiento económico de Bolivia y la tasa de Interés activa en moneda extranjera. Sin embargo, al modelar el Panel Dinámico, aparecen dos variables adicionales que son significativas para la cartera total como ser: el crédito comercial y la inercia de la cartera total. Cabe hacer notar que esta última variable no había sido incluida en la hipótesis original, pero su influencia era ya previsible de manera intuitiva, debido a que acciones pasadas de una variable $X$ tienden a afectar a la misma variable $X$ en periodos futuros, que en este caso sería la misma cartera total.

En ese sentido, el estudio detallado de los determinantes de la cartera total en el sistema microfinanciero en Bolivia es un tema nuevo, pero a la vez fascinante y por lo mismo debe ser una tarea permanente para el quehacer científico, pues el entendimiento de dichos determinantes, es una condición indispensable para lograr la adecuada instrumentación de una política financiera que vaya a favor de la sociedad misma. Por tanto, el presente documento no pretende llegar a una conclusión definitiva, sino más bien es una muestra del compromiso de analizar continuamente y en detalle el tema en cuestión.

\section{Bibliografía}

Caballero Benigno (2003), Notas y lecturas de clase de econometría, FCEFA, Universidad Técnica de Oruro.

Cameron, C. y P. Trivedi (2005), Microeconometrics-Methods and Applications, usA: Cambridge-Press.

Comité de Coordinación de Microfinanzas (2000), Marco integral de políticas para el y microempresa de la micro pequeña empresa, Fundapro.

González Vega, Claudio y Prado Guachalla (2001), El reto de las micro finanzas en América Latina, Fundapro. 
González Vega, Claudio y Rodriguez Meza, Jorge (2004), Las microfinanzas en el desarrollo del sistema financiero de Bolivia, Proyecto Premier, The Ohio State University.

Gujarati, Damodar, (2005), Econometría básica, México, DF, McGraw Hill.

Greene, William, (1998), Análisis econométrico, Madrid: Prentice-Hall.

Dai-Sefir (2002), La situación macroeconómica y el sector de las microfinanzas en Bolivia.

Economist Intellingence Unit. (2008), Microscopio 2008 sobre el entorno de negocios para las microfinanzas en América Latina y el Caribe.

Galindo, Luis y Catalán, Horacio (2003), La Tasa de Interés Real en México: un análisis de raíces unitarias con cambio estructural, Facultad de Economía, unAm. Johnston, J. y J. Dinardo (2001), Métodos de econometría, Barcelona: Vicens Vices. Loria, Eduardo (2009), Econometría con aplicaciones, Facultad de Economía, unAM.

Mendoza Miguel y Quintana Luis, (2008), Modelos y aplicaciones para la economía mexicana, Econometría básica. Facultad de Economía-unAM.

Navajas Sergio, Mark Schreiner, Richard L. Meyer, Gonzalez Vega Claudio y Jorge Rodriguez (2000), Microcredit and the Poorest of the Poor: Theory and Evidence from Bolivia, World Development.

Sánchez Armando, Spanos Aris y López Julio (2005), Macroeconomic linkages in México: A keynesian-structuralist perspective, Facultad de Economía, unAm.

Pindyck, R. S. y D. L. Rubinfeld (2001), Econometría. modelos y pronósticos. México: McGraw-Hill.

Ynaraja Ramírez, Ramón (2000), "Una experiencia en microfinanzas en Bolivia", Boletín Económico de ICE, núm. 2645.

Wooldridge, Jeffrey (2001), Econometric Analysis of Cross Section and Panel, USA: Mit-Press. 


\section{Anexo 1 \\ Pruebas de diagnóstico para el modelo de efectos fijos (cuadro 1)}

\section{a) Prueba de Autocorrelación Serial}

Prueba de Wooldridge de Autocorrelación en Datos Panel

Ho: No existe autocorrelación serial en el modelo de efectos fijos

Ha: Existe autocorrelación serial en el modelo de efectos fijos

Estadístico $\mathrm{F}(1,6)=3.360$

Prob. $>\mathrm{F}=0.1165$

Como la probabilidad es $0.1165>0.10$ se acepta la Ho, por lo tanto se concluye que no existe autocorrelación serial en el modelo de efectos fijos.

b) Prueba de Autocorrelación contemporáneo

(Matriz de Correlación de los Residuales)

\begin{tabular}{|c|r|r|r|r|r|r|l|}
\hline & e1 & e2 & e3 & e4 & e5 & e6 & e7 \\
\hline e1 & 1.0000 & & & & & & \\
\hline e2 & 0.8606 & 1.0000 & & & & & \\
\hline e3 & 0.6774 & 0.7095 & 1.0000 & & & & \\
\hline e4 & 0.1090 & 0.0752 & 0.1743 & 1.0000 & & & \\
\hline e5 & -0.0411 & -0.0596 & -0.2789 & -0.2624 & 1.0000 & & \\
\hline e6 & -0.4553 & -0.4572 & -0.2819 & 0.1123 & -0.3199 & 1.0000 & \\
\hline e7 & 0.2358 & 0.2195 & 0.3214 & -0.0199 & 0.5759 & -0.0598 & 1.0000 \\
\hline
\end{tabular}

Prueba de Independencia de LM Breusch-Pagan: chi2(21) = 30.563, Probabilidad $(\operatorname{Pr})=0.0813$

Ho: No existe autocorrelación contemporánea en el modelo de efectos fijos. Ha: Existe autocorrelación contemporánea en el modelo de efectos fijos.

Como la probabilidad $(\mathrm{Pr})$ es $=0.0813>0.05$ se acepta la Ho, por lo tanto se concluye que no existe correlación contemporánea en el modelo de efectos fijos. 


\section{c) Prueba de heterocedasticidad}

Ho: No existe heterocedasticidad en el modelo de efectos fijos.

Ha: Existe heterocedasticidad en el modelo de efectos fijos.

Prueba Modificada de Wald para Grupo Heterocedasticos

Estadístico chi $^{2}(7)=61.56$

Valor de Probabilidad $=0.0000$

La probabilidad es $=0.0000<0.10$, acá se acepta la Ha que existe varianza heterocedastica en los errores, es decir, que el modelo de efectos fijos tiene problemas de heterocedasticidad.

\section{d) Prueba de normalidad de los Residuos}

Ho: Existe normalidad en los residuos en el modelo de efectos fijos.

Ha: No existe normalidad en los residuos en el modelo de efectos fijos.

Como el valor de probabilidad del test de Jarque Bera es $0.2221>0.10$ y el valor de probabilidad del test de $\mathrm{Chi}^{2}$ es $0.15499>0.10$, se acepta la Ho, por lo tanto existe normalidad en los residuos en el modelo de efectos fijos y por ende se da luz verde para la realización de pruebas de hipótesis e inferencia en el modelo de efectos fijos.

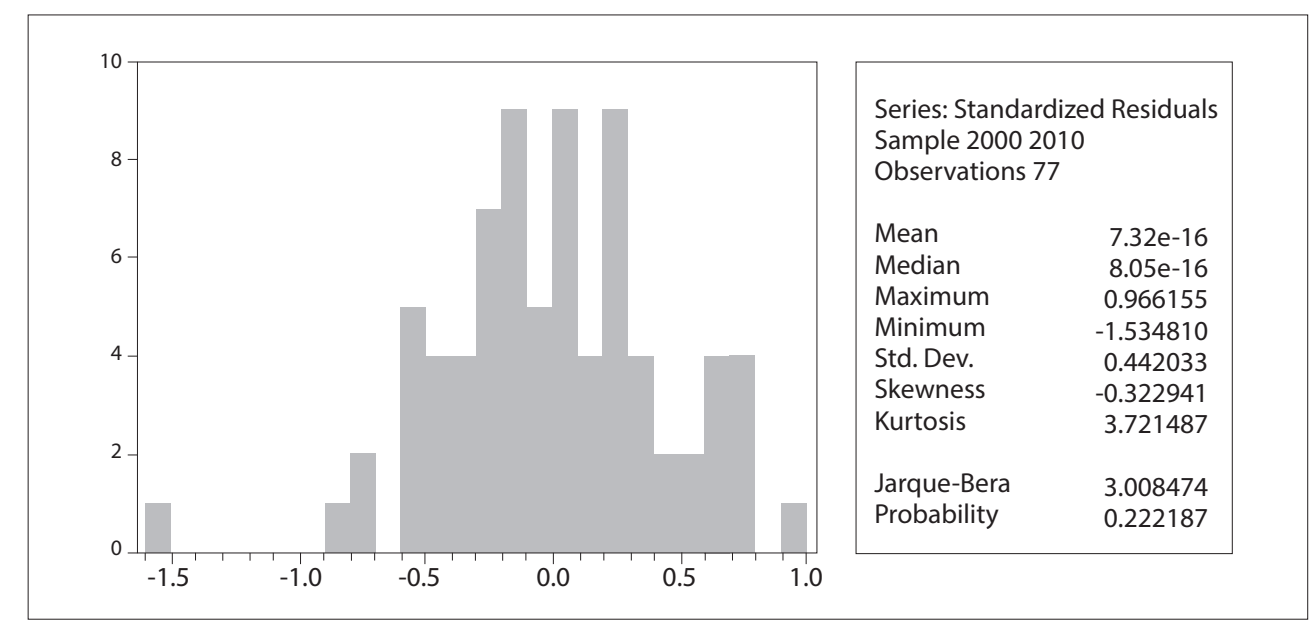



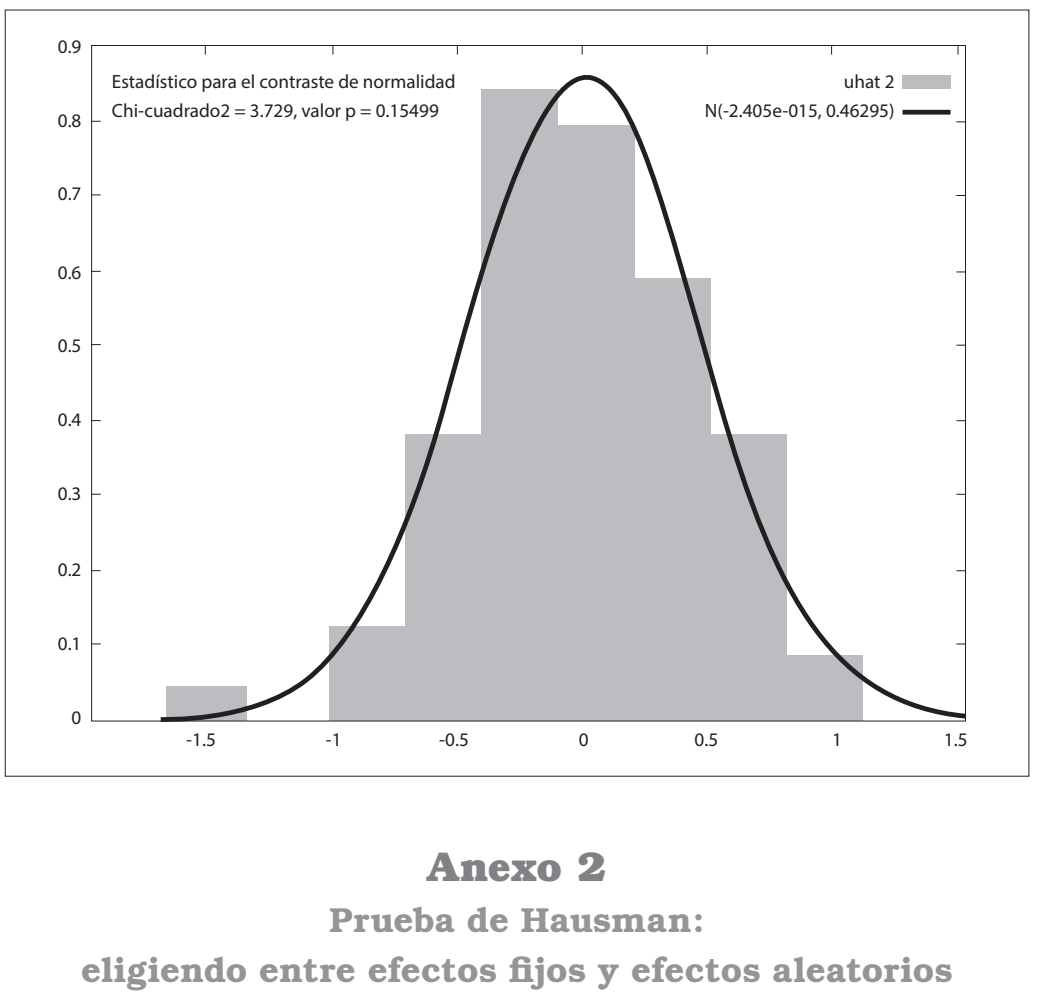

Esta prueba permite determinar que modelo es el más adecuado para el panel de datos que se está analizando, si el de efectos fijos o de efectos aleatorios. ${ }^{10}$

Utiliza para ello una prueba $\mathrm{Chi}^{2}$ con la hipótesis nula (Ho) de que el modelo de efectos aleatorios es el que mejor explica la relación de la variable dependiente con las explicativas, y por tanto se tiene la hipótesis alternativa (Ha) de que el mejor método que se ajusta es el de efectos fijos, por lo tanto la hipótesis es de la siguiente forma:

Ho: El modelo de efectos Aleatorios es mejor.

Ha: El modelo de Efectos Fijos es mejor.

Prueba de Hausman

$$
\begin{aligned}
\operatorname{chi}^{2}(7)= & (b-B)^{\wedge}\left[\left(V_{-} b-V_{-} B\right)^{\wedge}(-1)\right](b-B) \\
& \text { Estadístico }=4.96
\end{aligned}
$$

Valor de probabilidad $=0.0316$

Como la Prob. $=0.0316<0.10$ es significante, por lo tanto estadísticamente se acepta la Ha y se escoge al modelo de efectos fijos como aquel modelo panel que es más consistente.

\footnotetext{
${ }^{10}$ Véase Hausman, J., McFadden, C. (1984), "Specification test in econometrics", Econometrica, 52, 1219-1240. Alternativamente véase Greene, W.H. (1998), Análisis Econométrico, Madrid, Prentice-Hall, p. 112.
} 